\title{
ARTICLE
}

Received 26 Mar 2015 | Accepted 17 Jul 2015 | Published 26 Aug 2015

DOI: $10.1038 /$ ncomms9099 OPEN

\section{Dual-topology insertion of a dual-topology membrane protein}

Nicholas B. Woodall ${ }^{1}$ Ying Yin ${ }^{1} \&$ James U. Bowie ${ }^{1}$

Some membrane transporters are dual-topology dimers in which the subunits have inverted transmembrane topology. How a cell manages to generate equal populations of two opposite topologies from the same polypeptide chain remains unclear. For the dual-topology transporter EmrE, the evidence to date remains consistent with two extreme models. A post-translational model posits that topology remains malleable after synthesis and becomes fixed once the dimer forms. A second, co-translational model, posits that the protein inserts in both topologies in equal proportions. Here we show that while there is at least some limited topological malleability, the co-translational model likely dominates under normal circumstances.

\footnotetext{
${ }^{1}$ Department of Chemistry and Biochemistry, UCLA-DOE Institute, Molecular Biology Institute, University of California, Los Angeles, Los Angeles, California 90095-1570, USA. Correspondence and requests for materials should be addressed to J.U.B. (email: bowie@mbi.ucla.edu).
} 
A dual-topology protein presents a difficult problem for evolution to solve. How can the same polypeptide achieve equal populations of the two topologies? If one orientation is strongly preferred it will lead to many orphan subunits that do not have an opposite topology partner. A possible solution to this problem would be for topology to remain malleable after synthesis so that the topology is set only after an opposite topology partner is found, driven by the stability of the antiparallel dimer ${ }^{1}$. We will refer to this as post-translational dual-topology generation (Fig. 1a). A number of studies have shown that membrane protein topology can be altered after synthesis. In particular, the Skach lab showed that aquaporin-1 can insert in a partially incorrect topology and then re-orient after synthesis ${ }^{2}$ and work from the Dowhan group revealed that altering the lipid composition leads to a remarkable topology rearrangement of an entire domain of lactose permease $\mathrm{e}^{3,4}$.

The most well studied dual-topology transporter is the multidrug resistance protein $\mathrm{EmrE}^{1,5,6}$. In one subunit the $\mathrm{N}$ and $\mathrm{C}$ termini are inside the cytoplasm $\left(\mathrm{N}_{\text {in }} / \mathrm{C}_{\text {in }}\right)$ and in the other subunit, the $\mathrm{N}$ and $\mathrm{C}$ termini are outside $\left(\mathrm{N}_{\text {out }} / \mathrm{C}_{\text {out }}\right)^{7,8}$. The topology of helical membrane proteins can be predicted by the preference for positively charged amino acids to reside in the cytoplasm, the so-called positive-inside rule ${ }^{9,10}$. As a dual-topology protein, EmrE does not exhibit a positive-inside rule charge bias. By the strategic introduction of positive charges, however, it is possible to force subunits into either the $\mathrm{N}_{\text {in }} / \mathrm{C}_{\text {in }}$ topology or the $\mathrm{N}_{\text {out }} / \mathrm{C}_{\text {out }}$ topology ${ }^{11}$. Subunits forced into the $\mathrm{N}_{\text {in }} / \mathrm{C}_{\text {in }}$ topology will not form active dimers with themselves, but will form active dimers with subunits forced into the $\mathrm{N}_{\text {out }} / \mathrm{C}_{\text {out }}$ topology.
Studying the dual-topology protein EmrE, the von Heijne group showed that only subunits with $\mathrm{N}_{\text {out }} / \mathrm{C}_{\text {out }}$ topology would complement an EmrE construct with C-terminal positive charges ${ }^{12}$. These results have been interpreted to imply that topology can remain undefined until after the entire protein is made, consistent with the post-translational model (Fig. 1b) $)^{12-14}$. Yet these results can also be explained by a co-translational model in which the protein inserts in both topologies, but the C-terminal positive charges simply inactivate subunits initially inserted in the $\mathrm{N}_{\text {out }}$ topology (Fig. 1b). Consequently, without any direct examination of the inserted topologies, the indirect complementation experiments do not demonstrate topological malleability and the mechanism of dual-topology generation remains in question.

The problem with distinguishing between the post- and co-translational topology models is that the final state is the same: dual topology. What is needed is a way to lock the $\mathrm{N}$ terminus in its initially inserted topology. As described below, we find that placement of a short haemagglutinin (HA) epitope tag at the $\mathrm{N}$ terminus of the protein (HA-EmrE, Fig. 2a) allows the expression of both topologies of EmrE, but blocks subsequent topology rearrangement, allowing us to examine the co- and post-translational topology generation models. We find that some post-translational topology changes can occur, but that EmrE normally inserts in both $\mathrm{N}_{\text {in }}$ and $\mathrm{N}_{\text {out }}$ orientation with similar frequencies so that post-translational corrections are not a major mechanism for dual-topology insertion.

\section{Results}

HA tagging of EmrE does not prevent dual-topology insertion. We first asked whether the HA tag altered the functional behaviour

a
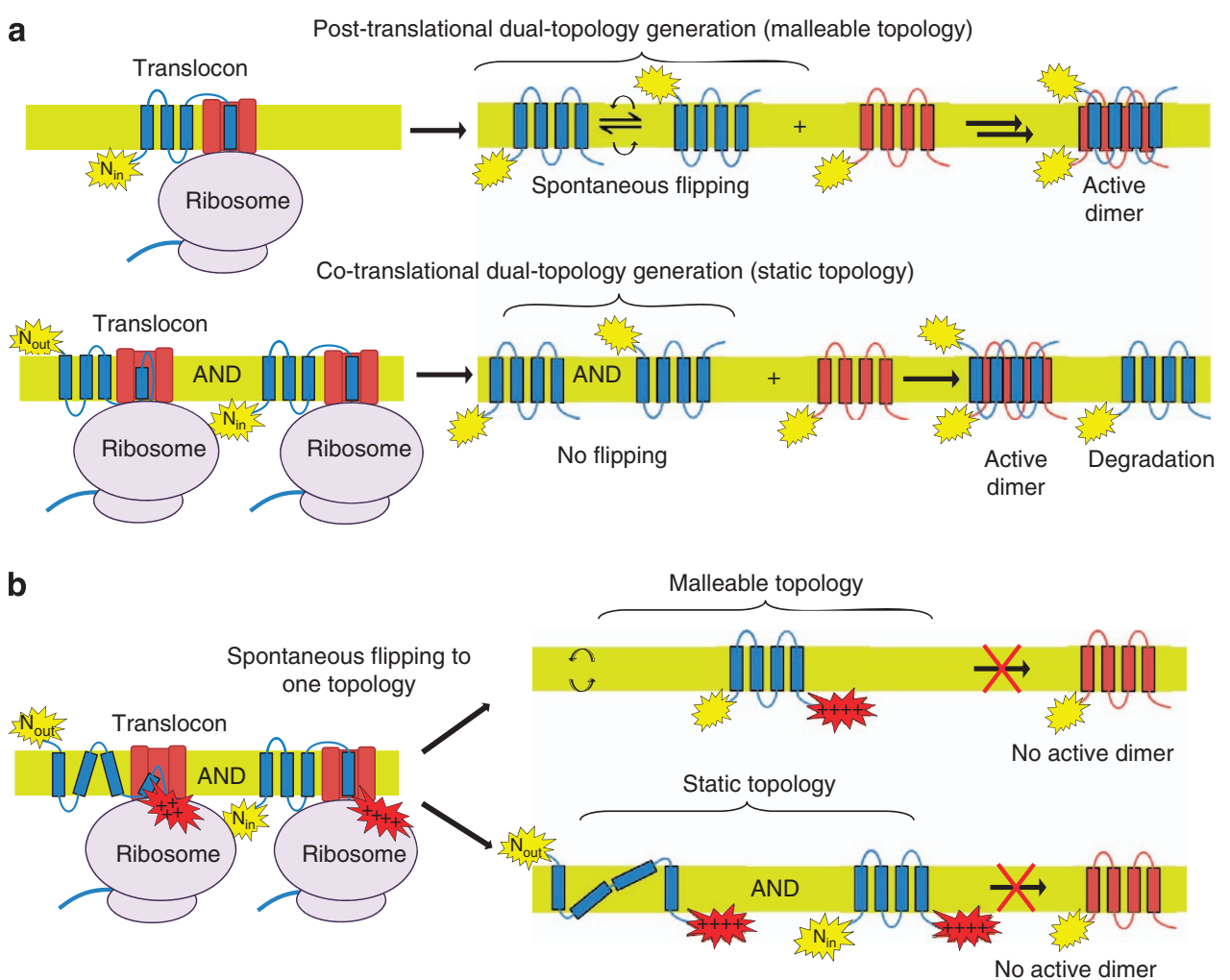

Figure 1 | Dual-topology insertion models. (a) Post-translational dual-topology model: EmrE inserts into the membrane in a preferred topology and then changes topology to form the stable antiparallel dimer. Co-translational dual-topology model: EmrE obtains dual topology by inserting equally into both topologies. Extra subunits are degraded. (b) Topology of EmrE with C-terminal positive charges if the $\mathrm{N}$-terminal helix has a malleable or static topology. Regardless of the mechanism, no active dimer can be formed with the $\mathrm{N}_{\text {in }} / \mathrm{C}_{\text {in }}$ EmrE. The frustrated $\mathrm{N}_{\text {out }} / \mathrm{C}_{\text {in }}$ topology shown is one of the many possible topologies. 
a

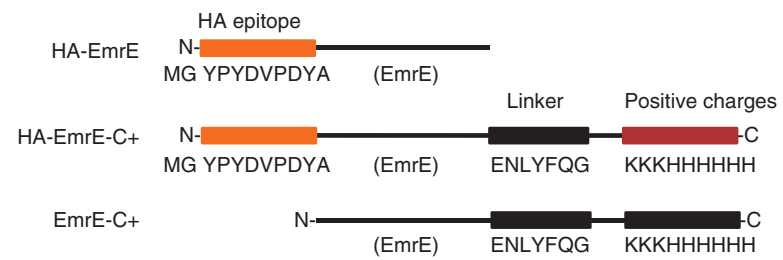

b

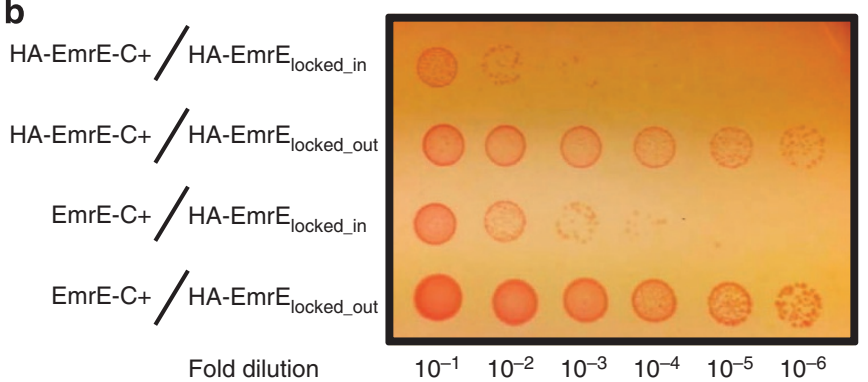

Figure 2 | Indirect topology assay of EmrE-C + and HA-EmrE-C + . (a) Diagram of the EmrE constructs used. (b) The growth of 10-fold dilutions of stationary phase cell culture expressing the HA-EmrE-C + or EmrE-C + constructs with a single topology EmrE mutant (HA-EmrE $E_{\text {locked_in }}$ or HA-EmrE locked_out $_{1}$ ), spotted on agar plates in the presence of $190 \mu \mathrm{g} \mathrm{ml}^{-1}$ ethidium bromide.

of the protein by attempting to recapitulate the prior in vivo results from the von Heijne group which showed that C-terminal positive charges would only generate active $\mathrm{N}_{\mathrm{in}} / \mathrm{C}_{\mathrm{in}}$ subunits ${ }^{12}$. As shown in Supplementary Fig. 1a, HA-EmrE imparts resistance to ethidium bromide suggesting that HA-EmrE is functional and can therefore attain a dual topology. We then created a construct with a cluster of positive charges (KKKHHHHHH) at the $\mathrm{C}$ terminus, HA-EmrE-C +, to mirror the construct used previously ${ }^{12}$ (Fig. 2a) and a control construct, EmrE-C+, without the HA tag, leaving a wild-type $\mathrm{N}$ terminus. We then tested the ability of these constructs to complement subunits forced into either the $\mathrm{N}_{\text {in }} / \mathrm{C}_{\text {in }}$ topology (HA-EmrE $\mathrm{E}_{\text {locked_in }}$ ) or the $\mathrm{N}_{\text {out }} / \mathrm{C}_{\text {out }}$ topology (HA-EmrE locked_out $_{\text {( }}$ by strategic placement of positive charges as described previously ${ }^{11}$. EmrE-C + and HA-EmrE-C + effectively complement the $\mathrm{N}_{\text {out }} / \mathrm{C}_{\text {out }}$ subunit HA-EmrE $E_{\text {locked_out }}$, but not

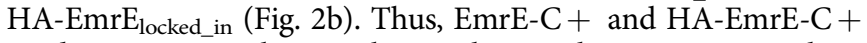
produce active subunits, but only in the $\mathrm{N}_{\text {in }} / \mathrm{C}_{\text {in }}$ topology, consistent with prior experiments ${ }^{12}$.

Rapid degradation of orphan EmrE subunits. We next sought to move beyond genetic complementation to directly map the topology of the constructs. We were initially stymied in these efforts, however, because both HA-EmrE-C + and EmrE-C+ had very low expression levels in the absence of the appropriate $\mathrm{N}_{\text {out }} / \mathrm{C}_{\text {out }}$ partner subunit. We therefore considered the possibility that orphan subunits might be rapidly proteolyzed. Figure $3 \mathrm{a}$ shows the degradation of HA-EmrE-C + over time in the presence of the incorrectly oriented subunit partner, HA-EmrE $E_{\text {locked in }}$ or in the presence of the correctly oriented subunit partner $\mathrm{HA}-\mathrm{EmrE}_{\text {locked_out. When dimer formation is }}$ precluded, the HA-EmrE-C + is degraded rapidly (half-life $<30 \mathrm{~min}$ ), while in the presence of the appropriate partner, there is essentially no detectable degradation after $120 \mathrm{~min}$. These results suggest that the $\mathrm{C}$-terminally charged constructs exist in an unstable form that is subject to proteolysis.

Blocking FtsH-mediated proteolysis of orphan EmrE subunits. $\mathrm{FtsH}$ is an integral membrane protease that is known to

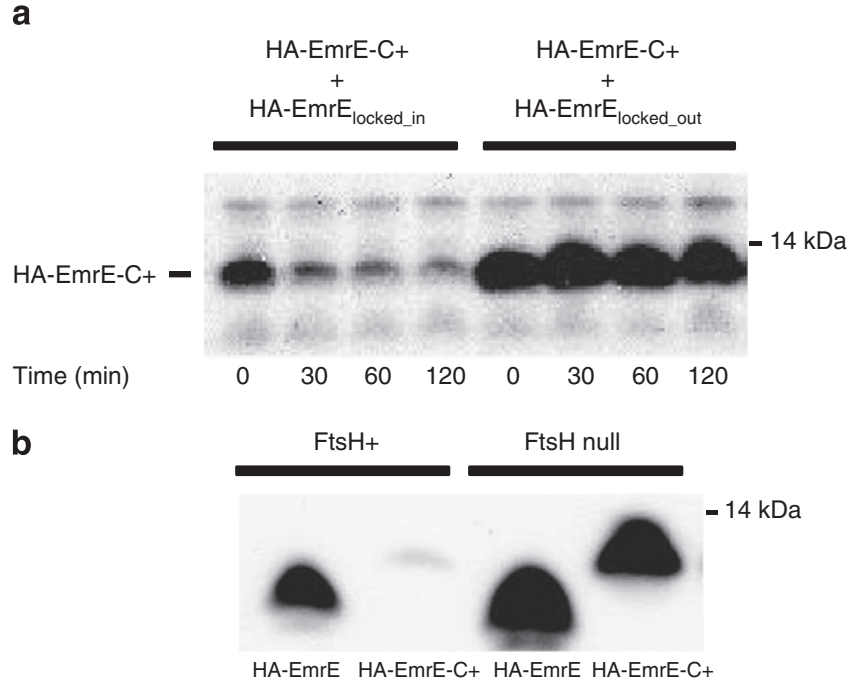

Figure 3 | FtsH-dependent proteolysis of EmrE. (a) The loss of HA-EmrE$\mathrm{C}+$ co-expressed with either HA-EmrE $\mathrm{I}_{\text {locked_in }}$ or HA-EmrE $\mathrm{E}_{\text {locked_out }}$ after protein synthesis has been stopped at time zero. HA-EmrE -C + was visualized by western blotting for the penta-histidine epitope. (b) The expression of HA-EmrE and HA-EmrE-C + in wild type and FtsH knockout cells. The protein was visualized by western blotting using the HA epitope.

preferentially degrade unstable membrane proteins ${ }^{15}$, so we tested whether FtsH was involved in degrading HA-EmrE-C + . Figure $3 \mathrm{~b}$ compares the expression levels in a control strain (AR3289) and an FtsH-null strain $(\mathrm{AR} 3291)^{16}$. Expression is $\sim 80$-fold higher in the FtsH-null strain than in the control strain, indicating that $\mathrm{FtsH}$ is at least partially responsible for the degradation of HA-EmrE-C + . The FtsH-null strain became an important tool for mapping the topological variants of EmrE, because it allowed us to map the topology of even unstable forms that might otherwise be lost to degradation.

With the ability to prevent proteolysis, we sought to directly determine the topology of EmrE-C + constructs using cysteine accessibility. Cysteine accessibility maps the topology of a membrane protein by identifying whether a single introduced cysteine resides in the cytoplasm or the periplasm, by measuring the reactivity of the cysteine to a membrane-impermeable reagent ${ }^{17}$. The procedure is outlined in Supplementary Fig. 2. We first add the membrane-impermeable reagent, 4 -acetamido- $4^{\prime}$ maleimidylstilbene-2,2'-disulfonic acid (AMS), and later a membrane-permeable biotinylation reagent, 3- $(N$-maleimidopropionyl)-biocytin (MPB). If AMS has already reacted with the lone cysteine (that is, cysteine has a periplasmic location) then reaction of MPB will be blocked by AMS. Otherwise MPB will modify the cysteine. The extent of MPB modification can be detected by a gel shift in the presence of avidin (seen as a loss of the gel band corresponding to free EmrE). To reliably assess the level of biotinylation without AMS, we also perform a reaction with MPB alone. To apply this method, we introduced unique cysteines (3C, F27C, Q81C and T108C) into a cysless background (C39A/ C41A/C95A), denoted as EmrE ${ }^{\text {Cless }}$. The EmrE ${ }^{\text {Cless }}$ variants were unreactive with MPB under our conditions and the intensity of the bands on western blots were linearly related to the amount of protein loaded (Supplementary Figs 3 and 4). We utilized the FtsH-null strain in all cysteine-accessibility topology experiments and three experimental replicates unless otherwise noted.

The topology of EmrE-C + is $\mathbf{N}_{\mathrm{in}} / \mathrm{C}_{\mathrm{in}}$. To assess the topology of EmrE ${ }^{\text {Cless }}-\mathrm{C}+$ with a wild-type $\mathrm{N}$ terminus, we mapped the 
a

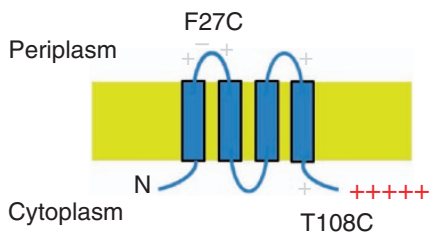

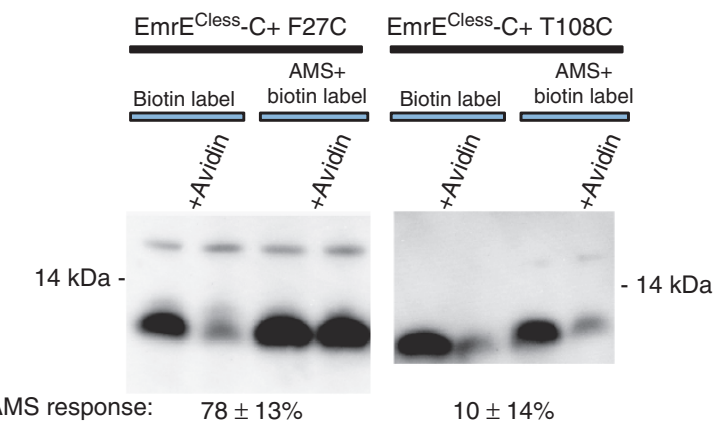

b

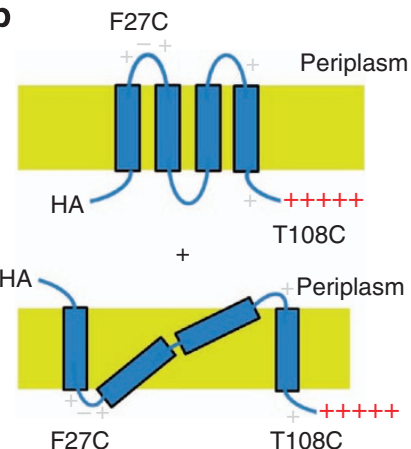

AMS response: $\quad 78 \pm 13 \%$

$10 \pm 14 \%$

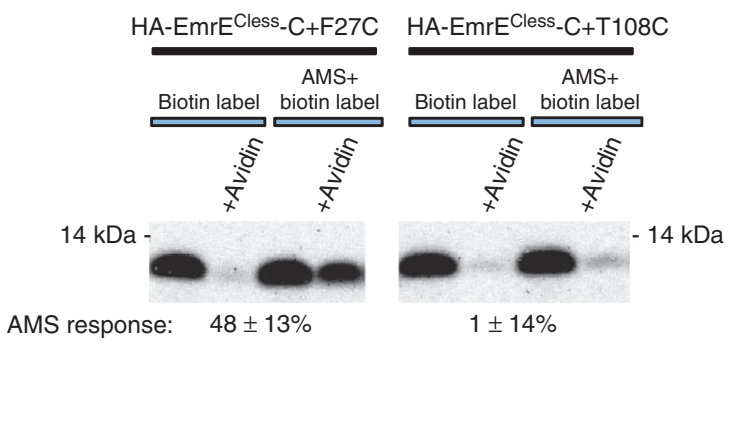

Figure 4 | Direct-topology assay of EmrE-C + and HA-EmrE-C +. Proposed topologies are shown on the left and cysteine-reactivity data are shown on the right. Added positively charged residues are shown in red. Invariant positively charged residues present in the wild-type protein are shown in light grey. AMS response is the per cent change in biotinylation due to the reaction with AMS. (a) Results for the EmrE ${ }^{\text {Cless }}$ - $C+$ constructs. The high AMS response of F27C $(n=4)$ is indicative of a periplasmic cysteine and the low AMS response of $\operatorname{T108C}(n=4)$ is indicative of a cytoplasmic location. (b) Results for the HA-EmrE-C + construct. While T108C $(n=3)$ shows a low AMS response similar to the EmrE ${ }^{C l e s s}-\mathrm{C}+$ construct that does not possess and HA tag, F27C $(n=5)$ shows much lower AMS response than seen in the EmrE ${ }^{\text {Cless }}-C+$ construct. EmrE ${ }^{\text {Cless }}-C+$ was visualized by western blotting for the pentahistidine epitope and HA-EmrE-C + was visualized by western blotting for the HA epitope.

locations of $\mathrm{F} 27 \mathrm{C}$ in the first loop and $\mathrm{T} 108 \mathrm{C}$ on the $\mathrm{C}$ terminus. As expected for an $\mathrm{N}_{\text {in }} / \mathrm{C}_{\text {in }}$ topology, $\mathrm{F} 27 \mathrm{C}$ is periplasmic as indicated by the large decrease in the avidin-dependent gel shift on prior reaction with AMS $(78 \pm 13 \%$ change compared with no AMS, error is standard deviation observed for $n=4$ replicates) (Fig. 4a). The C-terminally located T108C showed no change in gel shift after reaction with AMS (10 $\pm 14 \%$, $n=4$ ) indicating a cytoplasmic location, as is expected for an $\mathrm{N}_{\mathrm{in}} / \mathrm{C}_{\text {in }}$ topology (Fig. 4a). These results indicate that the $\mathrm{C}$-terminal positive charges do define the topology of the $\mathrm{N}$ terminus as suggested previously based on complementation experiments ${ }^{12}$.

Topology of HA-EmrE-C + . Continuing our characterization of the HA-tagged protein, we examined the topology of HA-EmrE ${ }^{\text {Cless }}-\mathrm{C}+$ using the same F27C and T108C mutations. A cysteine introduced into the first loop (F27C) of HAEmrE ${ }^{\text {Cless }}-\mathrm{C}+$ showed only partial blocking $(48 \pm 13 \%, n=5)$ by AMS (Fig. 4b). In contrast the C-terminal cysteine, T108C, which is adjacent to the $\mathrm{C}$-terminal positive charges showed no response to AMS $(1 \pm 4 \%, n=3)$, which indicates that it resides completely in the cytoplasm (Fig. 4b).

The partial reactivity of $\mathrm{F} 27 \mathrm{C}$ in the HA construct led us to consider the possibility that the $\mathrm{N}$ terminus normally inserts in two different topologies, but that the HA tag blocks subsequent topology rearrangement, leading to a mixed topology form as illustrated in Fig. 1b. We first had to evaluate other possible reasons for the partial reactivity, however.

Partial reactivity is not due to an environmental constraint. We considered the possibility that F27C is actually located fully in the periplasm in the HA-EmrE-C + construct, but is only partially reactive to AMS because it is partially buried by protein or membrane (even though this does not occur in the EmrE-C+ construct missing the HA tag). To test whether $\mathrm{F} 27 \mathrm{C}$ in HA-EmrE ${ }^{\text {Cless }}-\mathrm{C}+$ would be fully blocked by AMS if the protein were in a pure $\mathrm{N}_{\text {in }} / \mathrm{C}_{\text {in }}$ topology, we expressed the single-cysteine variants of $\mathrm{HA}-\mathrm{EmrE}^{\mathrm{Cless}}-\mathrm{C}+$ in a wild-type $\left(\mathrm{FtsH}^{+}\right)$strain in

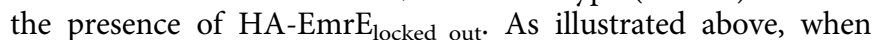
expressed in an $\mathrm{FtsH}^{+}$strain, the $\mathrm{N}_{\text {in }} / \mathrm{C}_{\text {in }}$ topology remains resistant to proteolysis when expressed in the presence of HA-EmrE $E_{\text {locked_out }}$ because it can form stable dimers, but orphan, unpaired subunits, are rapidly degraded by FtsH. Thus, only the $\mathrm{N}_{\text {in }} / \mathrm{C}_{\text {in }}$ topology of HA-EmrE ${ }^{\text {Cless }}-\mathrm{C}+$ will be present, allowing us to test the cysteine's reactivity in a pure $\mathrm{N}_{\text {in }} / \mathrm{C}_{\text {in }}$ subunit in the active dimer. As shown in Fig. 5a, when expressed with

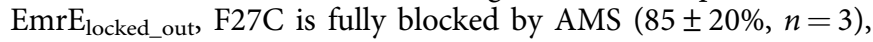
whereas T108C remained inaccessible to AMS $(5 \pm 6 \%, n=3)$. Moreover, Q81C, in the third loop was fully blocked by AMS ( $94 \pm 12 \%, n=3$ ), consistent with the $\mathrm{N}_{\mathrm{in}} / \mathrm{C}_{\mathrm{in}}$ topology when

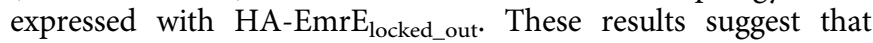
we could have seen complete blocking of the $\mathrm{F} 27 \mathrm{C}$ position if HA-EmrE ${ }^{\text {Cless }}-\mathrm{C}+$ adopted a pure $\mathrm{N}_{\text {in }} / \mathrm{C}_{\text {in }}$ topology.

It still remained possible that $\mathrm{F} 27 \mathrm{C}$ in $\mathrm{HA}-\mathrm{EmrE}^{\text {Cless }} \mathrm{C}+$ is more reactive to AMS in an active dimer compared with the orphan form in the absence of an $\mathrm{N}_{\text {out }} / \mathrm{C}_{\text {out }}$ subunit partner. To test this possibility, we attempted to generate pure normal $\mathrm{N}_{\text {in }} / \mathrm{C}_{\text {in }}$ and distorted $\mathrm{N}_{\text {out }} / \mathrm{C}_{\text {in }}$ topology orphan subunits by controlling the topology of the N-terminal helix. To favour an $\mathrm{N}_{\text {out }}$ orientation, we introduced a positive charge bias into loop 1 using the mutations G26R and T28R, to produce $\mathrm{N}_{\text {out }}-\mathrm{HA}-\mathrm{EmrE}^{\mathrm{Cless}}-\mathrm{C}+$ (Fig. 5b). To favour an $\mathrm{N}_{\text {in }}$ orientation, we placed positive charges at the $\mathrm{N}$ terminus by changing the N-terminal sequence from MPNYIY to 
a

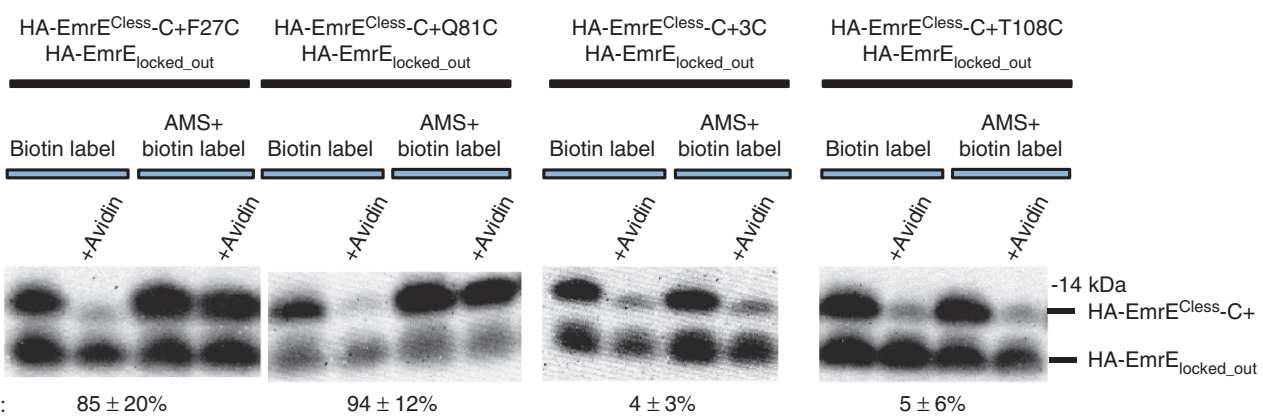

b

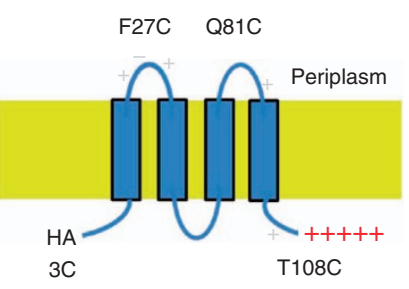

AMS response:
$85 \pm 20 \%$

$94 \pm 12 \%$

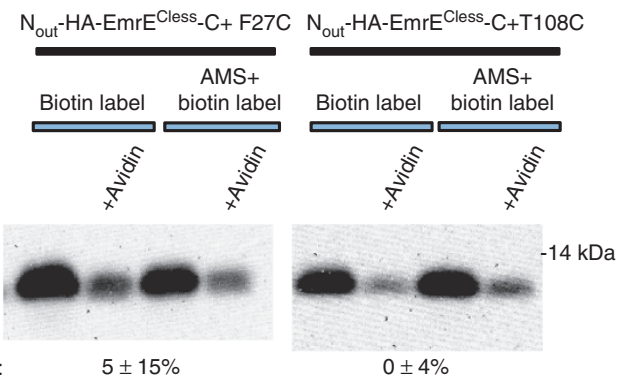

AMS response:

$5 \pm 15 \%$

$0 \pm 4 \%$

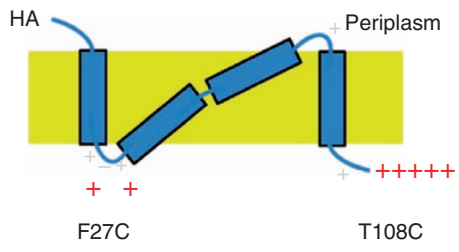

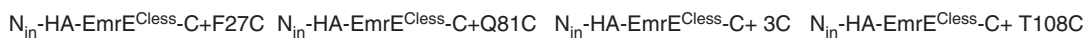

c

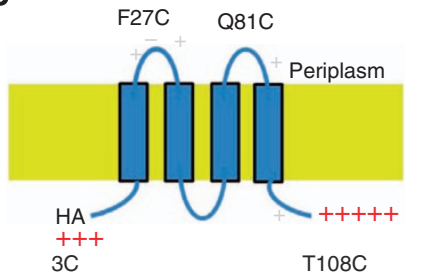

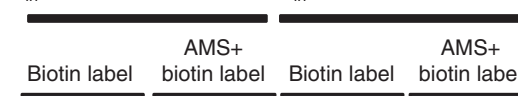

Biotin label biotin label Biotin label biotin label

AMS+
Biotin label
biotin labe

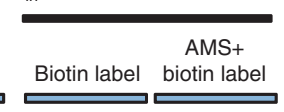
$\frac{x^{3}}{x^{5}}$
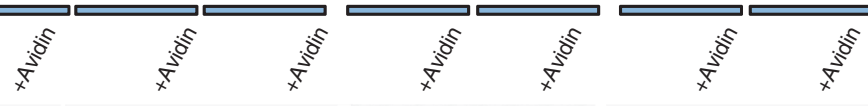

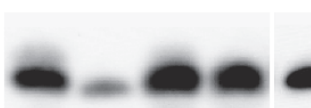

$78 \pm 10 \%$
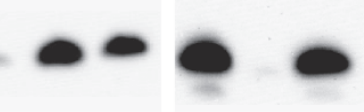

$61 \pm 7 \%$

$6 \pm 9 \%$

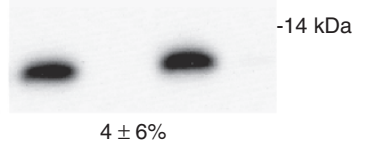

Figure 5 | AMS reactivity tests for EmrE constructs. Proposed topologies are shown on the left and cysteine-reactivity data are shown on the right. Added positively charged residues are shown in red. Invariant positively charged residues present in the wild-type protein are shown in light grey. AMS response is the per cent change in biotinylation due to the reaction with AMS. (a) AMS response of different cysteines in the HA-EmrECless-C + construct, when paired with $\mathrm{HA}-\mathrm{EmrE}_{\text {locked_out }}$ to generate an antiparallel dimer. Unpaired $\mathrm{HA}$-EmrE ${ }^{\text {Cless }}-\mathrm{C}+$ subunits are rapidly degraded by FtsH. (b) Topology mapping for the construct $\mathrm{N}_{\text {out }}-\mathrm{HA}-$ EmrE $\mathrm{Cless}_{-} \mathrm{C}+$. The frustrated $\mathrm{N}_{\text {out }} / \mathrm{C}_{\text {in }}$ topology shown is one of the many possible topologies. (c) Topology mapping for the construct

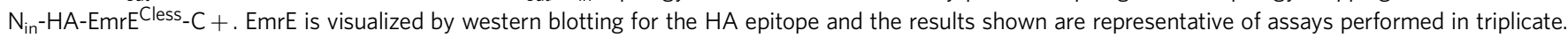

MRRRYIY, generating $\mathrm{N}_{\text {in }}$-HA-EmrE ${ }^{\text {Cless }}-\mathrm{C}+$ (Fig. 5c). Because of the introduced charge biases, the $\mathrm{N}$-terminal helix in these constructs should insert in unique orientations. Indeed for $\mathrm{N}_{\text {out }}$-HA-EmrE ${ }^{\text {Cless }}$ - $\mathrm{C}+$ construct, F27C and T108C no longer show any reaction to the AMS reagent $(5 \pm 15 \%$ and $0 \pm 4 \%$, respectively, $n=3$ ) indicating that $\mathrm{F} 27 \mathrm{C}$ and $\mathrm{T} 108 \mathrm{C}$ are cytoplasmic (Fig. 5b). Conversely, for $\mathrm{N}_{\text {in }}-\mathrm{HA}-\mathrm{EmrE}^{\text {Cless }}-\mathrm{C}+$, the $\mathrm{F} 27 \mathrm{C}$ position is nearly fully blocked by AMS $(78 \pm 10 \%, n=3)$ and $3 \mathrm{C}$ is completely unblocked $(6 \pm 9 \%, n=3)$, consistent with an $\mathrm{N}_{\text {in }}$ topology (Fig. 5c). Q81C shows a less complete AMS response $(61 \pm 7 \%, n=3)$, possibly due to an altered conformation in the monomeric subunit. Nevertheless, these results strongly suggest that the partial blockage observed for F27C in HA-EmrE ${ }^{\text {Cless }}-\mathrm{C}+$ is not due to the changes in reactivity of F27C.

We were unable to use cysteine accessibility to map the location of $3 \mathrm{C}$ in the HA-EmrE ${ }^{\text {Cless }}-\mathrm{C}+$ and $\mathrm{N}_{\text {out }}-\mathrm{HA}-\mathrm{EmrE}^{\text {Cless }}{ }_{-} \mathrm{C}+$ constructs because of disulfide bond formation of $3 \mathrm{C}$. As shown in Fig. 6, a band at a dimer molecular weight is seen in constructs where $3 \mathrm{C}$ is expected to reside in the periplasm, but not in constructs where $3 \mathrm{C}$ is expected to reside in the cytoplasm. The dimer band is eliminated after reduction by $\beta$-mercaptoethanol $(\beta-\mathrm{ME})$. Although disulfide formation complicates cysteine-

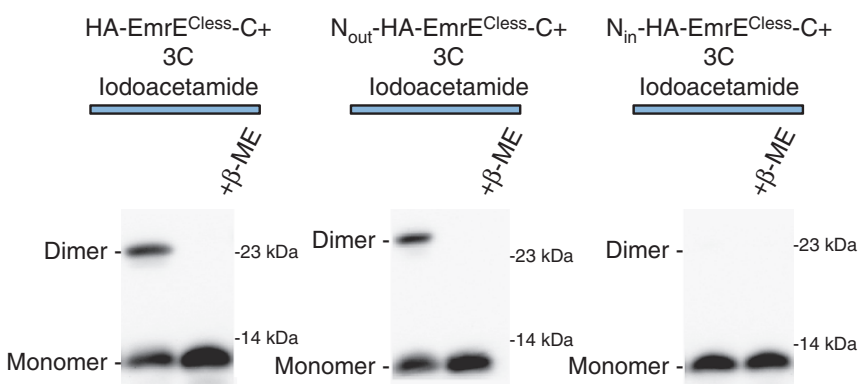

Figure 6 | Disulfide formation at the $\mathbf{N}$ terminus. A $3 C$ mutant in the context of the HA-EmrE $E^{\text {Cless }}-\mathrm{C}+$ and $\mathrm{N}_{\text {out }}-\mathrm{HA}-\mathrm{EmrE}^{\text {Cless }}-\mathrm{C}+$ form dimers that are sensitive to the addition of 2-mercaptoethanol suggest that the $3 \mathrm{C}$ can form disulfide-linked dimers. No dimers were seen for the $\mathrm{N}_{\mathrm{in}}-\mathrm{HA}$ EmrE ${ }^{\text {Cless }}-\mathrm{C}+$ construct. These results are consistent with a $3 \mathrm{C}$ location in the oxidizing periplasm for EmrE Cless $-\mathrm{C}+$ and $\mathrm{N}_{\text {out }}-\mathrm{HA}$-EmrE ${ }^{\text {Cless }}-\mathrm{C}+$ constructs and a $3 \mathrm{C}$ location in the reducing cytoplasm for the $\mathrm{N}_{\mathrm{in}}-\mathrm{HA}$ EmrE ${ }^{\text {Cless }}-\mathrm{C}+$ construct. Further disulfide formation was blocked by the addition of iodoacetamide prior to cell lysis. EmrE is visualized by western blotting for the HA epitope. 

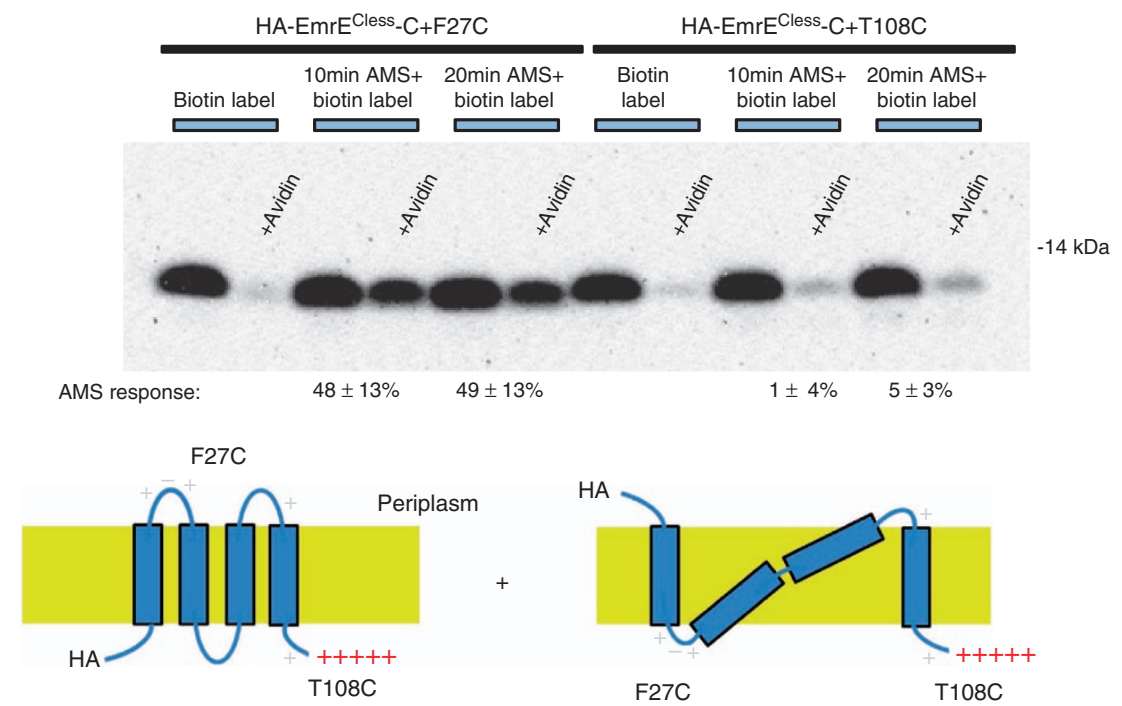

Figure 7 | Time dependence of AMS reactivity. The extent of AMS blocking of HA-EmrECless-C + F27C and T108C for 10 and 20 min is shown. Essentially no difference is observed, indicating that $10 \mathrm{~min}$ is sufficient to block all the available thiols in $\mathrm{F} 27 \mathrm{C}$ and that the extent of reaction reflects an end point rather than a slowed rate of modification. The experimental conditions are the same as described in Fig. 4. EmrE is visualized by western blotting for the HA epitope.

accessibility analysis, formation of the disulfide bond is indicative of an oxidizing, periplasmic location. In $\mathrm{N}_{\text {in }}-\mathrm{HA}-\mathrm{EmrE}^{\text {Cless }}-\mathrm{C}+$, the $3 \mathrm{C}$ cysteine has robust reactivity with MPB and does not form disulfide bonds as would be expected for its cytoplasmic location (Fig. 6). Thus, the observation of disulfide formation is consistent with the predicted topologies.

Partial reactivity is not due to topology flipping. We finally considered the possibility that the HA-tagged $\mathrm{N}$ terminus topology is not fixed in an $\mathrm{N}_{\text {in }}$ or $\mathrm{N}_{\text {out }}$ topology, but flips back and forth. If the N-terminal segment (including F27C) of $\mathrm{HA}-\mathrm{EmrE}-\mathrm{C}+$ can move from the cytoplasm to the periplasm, then the partial reactivity could be due to its presence in the periplasm for only a fraction of the time. If so, the extent of F27C blocking should be time dependent. When we increased the AMS reaction time with HA-EmrE ${ }^{\text {Cless }}-\mathrm{C}+\mathrm{F} 27 \mathrm{C}$, however, we saw no increase in AMS blocking. When blocked for 10 or 20 min we observed AMS blocking of $48 \pm 13 \%(n=3)$ and $49 \pm 13 \%(n=3)$, respectively (Fig. 7). These results are consistent with a static topology of the inserted HA-EmrE-C + , that is, the protein is either $\mathrm{N}_{\mathrm{in}} / \mathrm{C}_{\text {in }}$ or $\mathrm{N}_{\text {out }} / \mathrm{C}_{\text {in }}$ and does not flip after insertion.

\section{Discussion}

Our results indicate that under normal circumstances EmrE inserts in two opposite topologies at roughly equal frequencies. In particular, with an $\mathrm{HA}$ tag at the $\mathrm{N}$ terminus that blocks subsequent topology flipping, we observe insertion in both an $\mathrm{N}_{\text {in }}$ and an $\mathrm{N}_{\text {out }}$ topology. As the initial insertion occurs in both topologies with roughly equal proportions, it suggests that the primary mechanism for EmrE dual-topology biogenesis is co-translational (Fig. 1a). While we cannot rule out global topology flipping in the wild-type protein as predicted by the post-translational mechanism, we only have direct evidence for more limited topology rearrangements when the protein is unnaturally inserted in a distorted topology. Indeed, to our knowledge our work is the first direct demonstration of topological malleability in EmrE since a static topology would produce the same results in the complementation assays used previously $^{12}$ (see Fig. 1b). Moreover, we find that incorrectly inserted or unpaired subunits can be cleared rapidly by proteolysis so a mechanism for ensuring equal populations of both topologies by subsequent topology flipping is not necessary. When topological signals are manipulated by the placement of positive charges at the $\mathrm{C}$ terminus, causing initial insertion in a distorted $\mathrm{N}_{\text {out }} / \mathrm{C}_{\text {in }}$ topology, the topology can indeed rearrange to an $\mathrm{N}_{\text {in }} / \mathrm{C}_{\text {in }}$ topology as previously suggested by von Heijne and co-workers ${ }^{12}$. It is fascinating and remarkable that even partial topological rearrangements are possible, although they are likely driven by distorted topologies that may only rarely occur naturally. Thus, we suggest that major topology rearrangements are unlikely to play a major role in dual-topology generation with the normal EmrE topology signals.

\section{Methods}

Materials. AMS was purchased from Life Technologies (Grand Island, NY). MPB was purchased from Cayman Chemical (Ann Arbor, MI).

Plasmids and constructs. The EmrE constructs were cloned into the $\mathrm{pBAD} / \mathrm{His} \mathrm{A}$ plasmid (Invitrogen) using the NcoI and XhoI cut sites, which removes the built-in tags. For the co-expression experiments, the $\mathrm{EmrE}_{\text {locked_out }}$ and $\mathrm{EmrE}_{\text {locked_in }}$ constructs were cloned into another $\mathrm{pBAD}$ vector, which contains a chloramphenicolresistance gene and a ClodF13-derived CDF replicon. This vector was created from the pSEL1 vector described previously ${ }^{18}$. The CDF origin was removed from pCDFDuet-1 vector (Novagen) using the XbaI and NheI restriction sites and was ligated into the pSEL1 vector replacing the p15A origin using the same restriction sites. EmrE constructs were cloned into the vector using a NcoI and HindIII site. A second NcoI site in the chloramphenicol-resistant gene was removed by making a silent mutation of Thr172 from the ACC codon to the ACA codon using PCR quickchange mutagenesis.

HA-EmrE bears an N-terminal HA epitope (YPYDVPDYA) before the wildtype Escherichia coli (E. coli) EmrE sequence with an additional glycine downstream of the initial methionine. The amino acids KKKHHHHHH were added onto the $\mathrm{C}$ terminus for the constructs HA-EmrE-C + after a linker (ENLYFQG). The EmrE-C + construct has the same protein sequence as HA-EmrE-C + except with a wild-type $\mathrm{N}$ terminus. A more detailed view of these constructs is given in Supplementary Fig. 5.

Surprisingly, HA-EmrE can be induced at full induction in the pBAD system without the cellular toxicity normally associated with the overexpression of EmrE $^{11}$. Expression of the EmrE-C + construct lacking the HA tag was toxic in our pBAD system. We speculate that the reduced toxicity of HA-EmrE may be due to a predicted RNA hairpin accidentally included in the HA tag that reduces expression (Supplementary Fig. 5). To use EmrE-C + in our system, we therefore engineered a predicted RNA hairpin into EmrE-C + by altering the DNA but not the protein sequence. The DNA mutations A-7T (in the pBad His A plasmid) and C6T prevent the toxic overexpression of EmrE-C + in our pBAD system 
(Supplementary Fig. 5C,D). The N-terminal portion of the wild-type-EmrE construct contains the same DNA sequence as EmrE-C + .

All of the point mutations were introduced by quickchange PCR mutagenesis. The HA-EmrE locked_out $_{\text {construct based on a previous work }}{ }^{11}$ contains the $\mathrm{N}$-terminal HA tag of HA-EmrE to prevent toxicity and the mutations T28R, L85R and R106A. The HA-EmrE locked_in $_{\text {C }}$ construct has the same HA tag to prevent toxicity and was also based on previous work ${ }^{11}$ but contains an additional mutation (M1K) to ensure complete topological locking in our system (M1K, R29G, R82S and $\mathrm{S} 107 \mathrm{~K})^{11}$

In all constructs used with cysteine-accessibility experiment $\left(\mathrm{N}_{\mathrm{in}}-\mathrm{HA}-\right.$ EmrE ${ }_{\text {Cless }} \mathrm{C}+, \mathrm{N}_{\text {out }}$-HA-EmrE ${ }^{\text {Cless }}-\mathrm{C}+, \mathrm{HA}-\mathrm{EmrE}^{\text {Cless }}-\mathrm{C}+$ and

$\mathrm{EmrE}^{\mathrm{Cless}}-\mathrm{C}+$ ), the native cysteine residues were changed to alanine (C39A, C41 A and C95A). The added cysteines F27C, Q81C and T108C were then singly introduced into each of the cysteine null constructs. The mutation $3 \mathrm{C}$ adds the amino acids 'CG' after the initial two N-terminal residues, $M G$, which are upstream of the HA epitope. To form the $\mathrm{N}_{\mathrm{in}}-\mathrm{HA}-\mathrm{EmrE}^{\text {Cless }}-\mathrm{C}+$ construct, the HAEmrE ${ }^{\text {Cless }}-\mathrm{C}+$ construct had the mutations N2R, P3R and additional arginine inserted between the two residues. To form the $\mathrm{N}_{\text {out }}-\mathrm{HA}-\mathrm{EmrE}^{\text {Cless }}-\mathrm{C}+$ construct, the HA-EmrE ${ }^{\text {Cless }}-\mathrm{C}+$ construct had the mutations G26R and T28R incorporated into sequence.

Ethidium bromide-resistance assay. E. coli BL21Pro (Addgene/ClonTech) cells with the relevant plasmids were grown to saturation $\left(\sim 10 \mathrm{~h}\right.$ at $\left.37^{\circ} \mathrm{C}\right)$ and then serially diluted 10 -fold six times. Five microlitres of each dilution was spotted onto plates with $0.2 \%$ arabinose, $34 \mu \mathrm{g} \mathrm{ml}^{-1}$ chloramphenicol, $100 \mu \mathrm{g} \mathrm{ml}^{-1}$ ampicillin, and the indicated concentration of EtBr. The plated cells were grown at $37^{\circ} \mathrm{C}$ for $18 \mathrm{~h}$.

Cysteine-accessibility topology assay. AR3291 (FtsH null) cells ${ }^{16}$ containing the desired EmrE construct were grown to $\sim 0.8 \mathrm{OD}_{600}$ and then induced with $0.2 \%$ arabinose at $30^{\circ} \mathrm{C}$ for $2 \mathrm{~h}$. Cells from $50 \mathrm{ml}$ of cell culture were collected by centrifugation and suspended in $500 \mu \mathrm{l}$ of $50 \mathrm{mM}$ phosphate buffer with $17 \mathrm{mM}$ $\mathrm{NaCl}$ at $\mathrm{pH}$ 8.0. Two-hundred microlitres of resuspended cells were incubated in a final concentration of $2 \mathrm{mM}$ AMS for $10 \mathrm{~min}$, rotating in the dark at room temperature. The cells were then washed twice with $50 \mathrm{mM}$ Tris- $\mathrm{Cl}$ at $\mathrm{pH}$ 7.5. The final cell pellet was resuspended to a final volume of $\sim 200 \mu$ l. Samples not reacted with AMS underwent the same procedure without the AMS reagent. The cells were further incubated with $4 \mathrm{mM}$ MPB in $4 \%$ dimethylsulphoxide, $0.5 \%$ toluene and $50 \mathrm{mM}$ Tris- $\mathrm{Cl}$ at $\mathrm{pH} 7.5$ for $1 \mathrm{~h}$ while rotating in the dark at room temperature. The cells were then lysed by sonication. Cell debris was removed by centrifugation at $16,000 \mathrm{~g}$ for $10 \mathrm{~min}$ and then membranes were isolated by ultracentrifugation of the supernatant in a Beckman Coulter Airfuge at $160,000 \mathrm{~g}$ for $1 \mathrm{~h}$ at room temperature. Membranes were resuspended in $20 \mathrm{mM}$ Tris- $\mathrm{Cl}$ at $\mathrm{pH}$ 7.5. Total protein was determined by the DC Protein Assay (Bio-Rad). For each lane, $24 \mu \mathrm{g}$ of protein was mixed with $4 \times$ SDS loading buffer. Either $25 \mu \mathrm{g}$ of avidin (Sigma BioUltra) in $20 \mathrm{mM}$ Tris-Cl at $\mathrm{pH} 7.5$ or $20 \mathrm{mM}$ Tris-Cl at $\mathrm{pH} 7.5$ was added to the samples and the samples were run on a $12 \%$ NuPAGE Bis-Tris (Life Technologies) gel using MES running buffer. The Accuruler Prestained Protein Ladder was used to estimate molecular weight. Electrophoresis was carried out at $40 \mathrm{~V}$ for $25 \mathrm{~min}$ and then $100 \mathrm{~V}$ for $135 \mathrm{~min}$. The samples were then transferred to a $(0.2 \mu \mathrm{m})$ PVDF membrane at $95 \mathrm{~mA}$ for $1 \mathrm{~h}$ using Towbin transfer buffer with $20 \%$ methanol.

For the HA antibody, the membrane was blocked with $5 \%$ non-fat milk in TBS-T ( $20 \mathrm{mM}$ Tris-Cl at pH 7.5, $150 \mathrm{mM} \mathrm{NaCl}, 0.1 \%$ Tween 20 ) followed by an hour incubation in a 1:1,000 dilution of $1 \mathrm{mg} \mathrm{ml}^{-1}$ monoclonal HA antibody (Sigma \#H3663) in TBS-T with $0.5 \%$ non-fat milk. The blot was then washed four times in $20 \mathrm{mM}$ Tris- $\mathrm{Cl}$ at $\mathrm{pH} 7.5,500 \mathrm{mM} \mathrm{NaCl}, 0.2 \%$ Triton X-100 and $0.05 \%$ Tween 20 buffer (TBS-Tween/Triton). The blot was incubated for $1 \mathrm{~h}$ in a 1:3,000 dilution of anti-mouse IgG peroxidase conjugate (Sigma \#A4416) in TBS-T with $0.5 \%$ non-fat milk and then washed six times for $10 \mathrm{~min}$ in TBS-Tween/Triton buffer. Western blots for the histidine tag were performed as described above except that the membrane was blocked and blotted with the supplied blocking buffer and the pentahis horseradish peroxidase conjugate antibody (Qiagen \#34460) as described in the QIAexpress detection and assay handbook (1:1,000 dilution).

The blots were visualized on a FluorChem FC2 (Alpha Innotech) CCD imager using the Amersham ECL Prime detection reagent (GE Healthcare) according to the recommended protocol. The intensity of the bands was quantified using ImageJ. A general background subtraction obtained from a dark area of the blot was applied to each band. The amount of biotinylation was determined by the decrease in band intensity between the lanes with and without avidin. The per cent change in biotinylation between the reaction with MPB only and the reaction with both AMS and MPB (labelled AMS response) was used to determine the location of each cysteine. An uncropped blot image is shown in Supplementary Fig. 6.

CCL EmrE degradation assay. BL21Pro E. coli cells co-expressing HAEmrE ${ }^{\text {Cless }}-\mathrm{C}+$ with either $\mathrm{N}_{\text {out }}-\mathrm{HA}-\mathrm{EmrE}^{\text {Cless }}-\mathrm{C}+$ or $\mathrm{N}_{\text {in }}-\mathrm{HA}-\mathrm{EmrE}^{\text {Cless }}-\mathrm{C}+$ were grown to $\sim 0.8 \mathrm{OD}_{600}$ and induced with $0.2 \%$ arabinose for $1 \mathrm{~h}$ at $30^{\circ} \mathrm{C}$. The cells were washed twice with LB media and then switched into LB media containing $350 \mu \mathrm{g} \mathrm{ml}^{-1}$ erythromycin and $0.2 \%$ glucose to stop further protein synthesis.
Samples were taken at time zero (after the media switch) and at the other indicated time points.

FtsH-null expression test. AR3291 cells (FtsH null) or AR3289 (control cells) obtained from the Ogura Lab ${ }^{16}$ were grown to $\sim 0.8 \mathrm{OD}_{600}$ and then induced with $0.2 \%$ arabinose for $2 \mathrm{~h}$ at $30^{\circ} \mathrm{C}$. The cells were collected by centrifugation and lysed by sonication. The membranes were isolated and the samples were visualized by western blot for the HA epitope as described previously.

Disulfide bond determination. AR3291 (FtsH null) cells containing the desired EmrE construct were grown to $\sim 0.8 \mathrm{OD}_{600}$ and then induced with $0.2 \%$ arabinose at $30^{\circ} \mathrm{C}$ for $2 \mathrm{~h}$. Cells from $50 \mathrm{ml}$ of cell culture were collected by centrifugation and suspended in $500 \mu \mathrm{l}$ of $50 \mathrm{mM}$ Tris-Cl buffer at $\mathrm{pH} 7.5$. Two-hundred microlitres of resuspended cells were incubated in a final concentration of $5 \mathrm{mM}$ ioacetamide for $15 \mathrm{~min}$, rotating in the dark at room temperature. The cells were then washed twice with $50 \mathrm{mM}$ Tris- $\mathrm{Cl}$ at $\mathrm{pH} 7.5$ and lysed by sonication. Cell debris was removed by centrifugation at $16,000 \mathrm{~g}$ for $10 \mathrm{~min}$ and then membranes isolated by ultracentrifugation of the supernatant in a Beckman Coulter Airfuge at $160,000 \mathrm{~g}$ for $1 \mathrm{~h}$ at room temperature. Isolated membranes were resuspended in $20 \mathrm{mM}$ Tris- $\mathrm{Cl}$ at $\mathrm{pH}$ 7.5. Twenty-four micrograms of the samples were then mixed in SDS loading buffer with 2-mercaptoethanol ( $\beta$-ME) at $170 \mathrm{mM}$ and without. The protein was visualized by western blot using the HA epitope as described earlier.

\section{References}

1. Lloris-Garcera, P. et al. Antiparallel dimers of the small multidrug resistance protein EmrE are more stable than parallel dimers. J. Biol. Chem. 287, 26052-26059 (2012).

2. Lu, Y. et al. Reorientation of aquaporin-1 topology during maturation in the endoplasmic reticulum. Mol. Biol. Cell 11, 2973-2985 (2000).

3. Vitrac, H., Bogdanov, M. \& Dowhan, W. In vitro reconstitution of lipiddependent dual topology and postassembly topological switching of a membrane protein. Proc. Natl. Acad. Sci USA 110, 9338-9343 (2013).

4. Bogdanov, M., Heacock, P. N. \& Dowhan, W. A polytopic membrane protein displays a reversible topology dependent on membrane lipid composition. EMBO J. 21, 2107-2116 (2002).

5. Lloris-Garcerá, P. et al. In vivo trp scanning of the small multidrug resistance protein EmrE confirms 3D structure models'. J. Mol. Biol. 425, 4642-4651 (2013).

6. Ubarretxena-Belandia, I., Baldwin, J. M., Schuldiner, S. \& Tate, C. G. Threedimensional structure of the bacterial multidrug transporter EmrE shows it is an asymmetric homodimer. EMBO J. 22, 6175-6181 (2003).

7. Rapp, M., Granset, E., Seppala, S. \& von Heijne, G. Identification and evolution of dual-topology membrane proteins. Nat. Struct. Mol. Biol. 13, 112-116 (2006).

8. Nasie, I., Steiner-Mordoch, S., Gold, A. \& Schuldiner, S. Topologically random insertion of EmrE supports a pathway for evolution of inverted repeats in ioncoupled transporters. J. Biol. Chem. 285, 15234-15244 (2010).

9. Von Heijne, G. The distribution of positively charged residues in bacterial inner membrane proteins correlates with the trans-membrane topology. EMBO J. 5, 3021 (1986).

10. Von Heijne, G. Membrane protein structure prediction hydrophobicity analysis and the positive-inside rule. J. Mol. Biol. 487-494 (1992).

11. Rapp, M., Seppala, S., Granseth, E. \& von Heijne, G. Emulating membrane protein evolution by rational design. Science 315, 1282-1284 (2007).

12. Seppala, S., Slusky, J. S., Lloris-Garcera, P., Rapp, M. \& von Heijne, G. Control of membrane protein topology by a single C-terminal residue. Science 328, 1698-1700 (2010).

13. Tate, C. Membrane protein gymnastics. Science 328, 1644-1645 (2010).

14. Bowie, J. Membrane protein twists and turns. Science 339, 398-399 (2013).

15. Herman, C., Prakash, S., Lu, C. Z., Matouschek, A. \& Gross, C. A. Lack of a robust unfoldase activity confers a unique level of substrate specificity to the universal AAA protease FtsH. Mol. Cell 11, 659-669 (2003).

16. Ogura, T. et al. Balanced biosynthesis of major membrane components through regulated degradation of the committed enzyme of lipid A biosynthesis by the AAA protease FtsH (HflB) in Escherichia coli. Mol. Microbiol. 31, 833-844 (1999).

17. Bogdanov, M., Zhang, W., Xie, J. \& Dowhan, W. Transmembrane protein topology mapping by the substituted cysteine accessibility method (SCAMTM): application to lipid-specific membrane protein topogenesis. Methods 36, 148-171 (2005).

18. Massey-Gendel, E. et al. Genetic selection system for improving recombinant membrane protein expression in E. coli. Protein Sci. 18, 372-383 (2009). 


\section{Acknowledgements}

This work was supported by a NIH Grant RO1 GM063919 to J.U.B. and an NIH Chemistry/Biology Interface Training Grant to N.B.W. We thank Teru Ogura for supplying the AR3289 and AR3291 strains.

\section{Author contributions}

N.B.W. and J.U.B. conceived the project and designed the experiments; N.B.W. and Y.Y. conducted experiments; N.B.W., J.U.B. and Y.Y. analysed data; N.B.W. and J.U.B. wrote the manuscript.

\section{Additional information}

Supplementary Information accompanies this paper at http://www.nature.com/ naturecommunications
Competing financial interests: The authors declare no competing financial interests.

Reprints and permission information is available online at http://npg.nature.com/ reprintsandpermissions/

How to cite this article: Woodall, N. B. et al. Dual-topology insertion of a dual-topology membrane protein. Nat. Commun. 6:8099 doi: 10.1038/ncomms9099 (2015).

(c) (i) This work is licensed under a Creative Commons Attribution 4.0 International License. The images or other third party material in this article are included in the article's Creative Commons license, unless indicated otherwise in the credit line; if the material is not included under the Creative Commons license, users will need to obtain permission from the license holder to reproduce the material. To view a copy of this license, visit http://creativecommons.org/licenses/by/4.0/ 